\title{
Galantamine alleviates inflammation and insulin resistance in patients with metabolic syndrome in a randomized trial
}

Fernanda M. Consolim-Colombo,, ${ }^{1,2}$ Carine T. Sangaleti, ${ }^{1,3}$ Fernando O. Costa, ${ }^{2}$ Tercio L. Morais, ${ }^{2}$ Heno F. Lopes, ${ }^{1,2}$ Josiane M. Motta, ${ }^{2}$ Maria C. Irigoyen, ${ }^{1}$ Luiz A. Bortoloto, ${ }^{1}$ Carlos Eduardo Rochitte, ${ }^{1}$ Yael Tobi Harris, ${ }^{4}$ Sanjaya K. Satapathy, ${ }^{5}$ Peder S. Olofsson, ${ }^{6,8}$ Meredith Akerman, ${ }^{9}$ Sangeeta S. Chavan, ${ }^{6,7}$ Meggan MacKay, ${ }^{10}$ Douglas P. Barnaby, ${ }^{11}$ Martin L. Lesser, ${ }^{9}$ Jesse Roth, ${ }^{12}$ Kevin J. Tracey, ${ }^{6,7}$ and Valentin A. Pavlov ${ }^{6,7}$

'University of Sao Paulo, Hypertension Unit, Sao Paulo, Brazil. ${ }^{2}$ Nove de Julho University (UNINOVE), PPG, Sao Paulo, Brazil. ${ }^{3}$ Midwestern State University (UNICENTRO), Paraná, Brazil. ${ }^{4}$ Hofstra Northwell School of Medicine at Hofstra University, Division of Endocrinology, Diabetes and Metabolism, Hempstead, New York, USA. ${ }^{5}$ Methodist University Hospital, University of Tennessee Health Sciences Center, Memphis, Tennessee, USA. ${ }^{6}$ Center for Biomedical Science, and ${ }^{7}$ Center for Bioelectronic Medicine, The Feinstein Institute for Medical Research, Northwell Health, Manhasset, New York, USA. ${ }^{8}$ Center for Bioelectronic Medicine, Department of Medicine, Center for Molecular Medicine, Solna, Karolinska Institutet, Karolinska University Hospital, Stockholm, Sweden. ${ }^{9}$ Biostatistics Unit, and ${ }^{10} \mathrm{Center}$ for Autoimmune \& Musculoskeletal Disease, The Feinstein Institute for Medical Research, Northwell Health, Manhasset, New York, USA. "Department of Emergency Medicine of Albert Einstein College of Medicine, Bronx, New York, USA. ${ }^{12}$ Laboratory of Diabetes and Diabetes-related Research, The Feinstein Institute for Medical Research, Northwell Health, Manhasset, New York, USA.

Role of funding source: This study was funded by Fundação de Amparo a Pesquisa do Estado de São Paulo (FAPESP), Conselho Nacional de Desenvolvimento Científico e Tecnológico (CNPq), and the $\mathrm{NIH}$, National Institute of General Medical Sciences. The funders of the study had no role in study design, data collection, data analysis, data interpretation, or writing the report.

\section{Authorship note: KJT and VAP} contributed equally to this work as co-senior authors

Conflict of interest: VAP and KJT have published patents (US 8,865,641 B2 and US 8,003,632 B2) broadly relevant to this work and have assigned their rights to the Feinstein Institute for Medical Research.

Submitted: February 8, 2017

Accepted: June 6, 2017

Published: July 20, 2017

\section{Reference information:}

JCI Insight. 2017;2(14):e93340.

https://doi.org/10.1172/jci.

insight. 93340.
BACKGROUND. Metabolic syndrome (MetS) is an obesity-driven condition of pandemic proportions that increases the risk of type 2 diabetes and cardiovascular disease. Pathophysiological mechanisms are poorly understood, though inflammation has been implicated in MetS pathogenesis. The aim of this study was to assess the effects of galantamine, a centrally acting acetylcholinesterase inhibitor with antiinflammatory properties, on markers of inflammation implicated in insulin resistance and cardiovascular risk, and other metabolic and cardiovascular indices in subjects with MetS.

METHODS. In this randomized, double-blind, placebo-controlled trial, subjects with MetS (30 per group) received oral galantamine $8 \mathrm{mg}$ daily for 4 weeks, followed by $16 \mathrm{mg}$ daily for 8 weeks or placebo. The primary outcome was inflammation assessed through plasma levels of cytokines and adipokines associated with MetS. Secondary endpoints included body weight, fat tissue depots, plasma glucose, insulin, homeostasis model assessment of insulin resistance (HOMA-IR), cholesterol (total, HDL, LDL), triglycerides, BP, heart rate, and heart rate variability (HRV).

RESULTS. Galantamine resulted in lower plasma levels of proinflammatory molecules TNF (-2.57 $\mathrm{pg} / \mathrm{ml}[95 \% \mathrm{Cl}-4.96$ to -0.19$] ; P=0.035)$ and leptin $(-12.02 \mathrm{ng} / \mathrm{ml}[95 \% \mathrm{Cl}-17.71$ to -6.33$] ; P<$ $0.0001)$, and higher levels of the antiinflammatory molecules adiponectin $(2.71 \mu \mathrm{g} / \mathrm{ml}[95 \% \mathrm{Cl}$ 1.93 to 3.49]; $P<0.0001)$ and IL-10 (1.32 pg/ml, [95\% Cl 0.29 to 2.38]; $P=0.002$ ) as compared with placebo. Galantamine also significantly lowered plasma insulin and HOMA-IR values, and altered HRV.

CONCLUSION. Low-dose galantamine alleviates inflammation and insulin resistance in MetS subjects. These findings support further study of galantamine in MetS therapy.

TRIAL REGISTRATION. ClinicalTrials.gov, number NCT02283242.

FUNDING. Fundação de Amparo a Pesquisa do Estado de São Paulo (FAPESP) and Conselho Nacional de Desenvolvimento Científico e Tecnológico (CNPq), Brazil, and the NIH. 


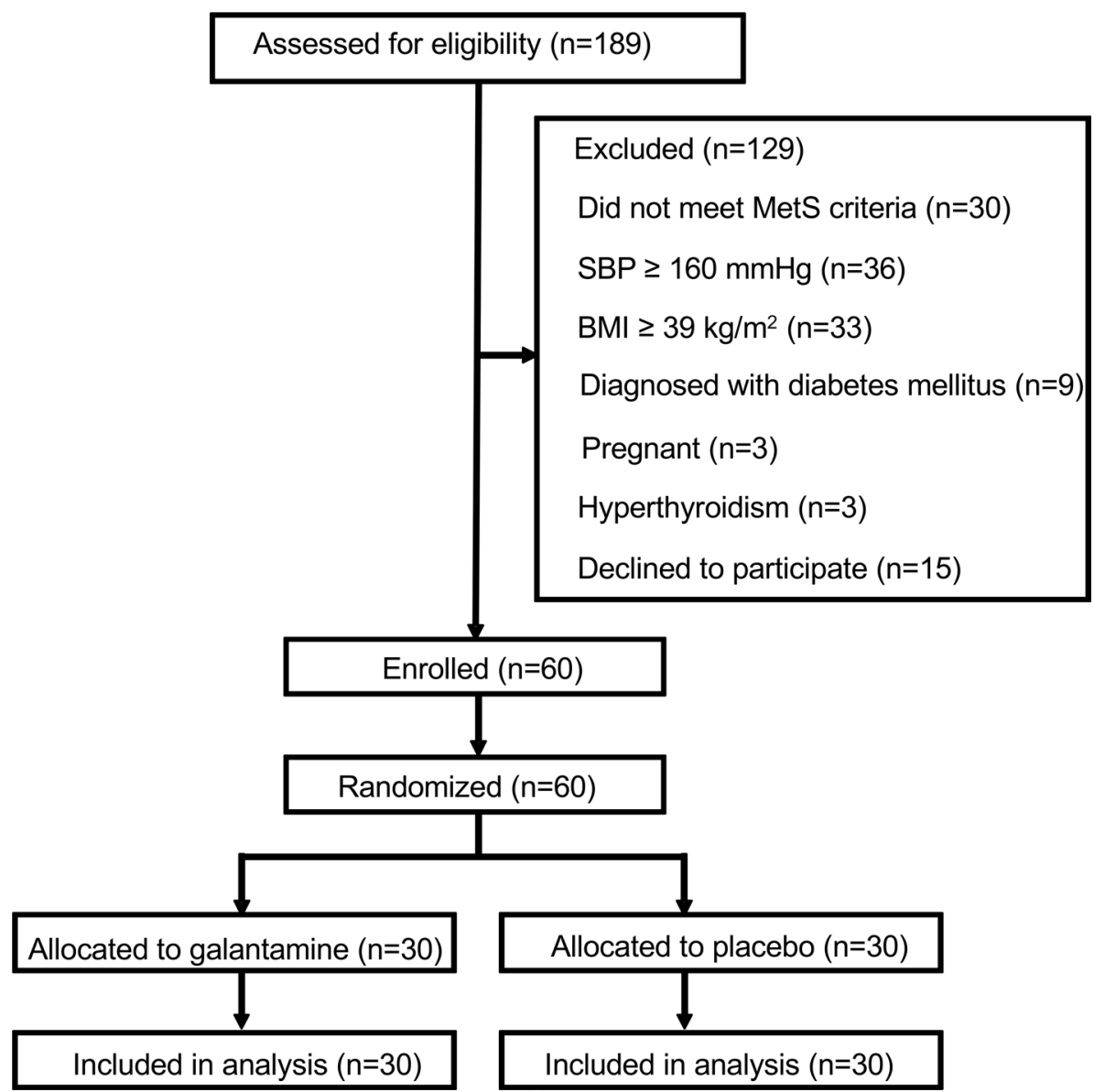

Figure 1. Trial consort flow diagram. MetS, metabolic syndrome; SBP, systolic BP.

\section{Introduction}

Central (abdominal) obesity, elevated fasting glucose, dyslipidemia (abnormally high triglycerides and low HDL cholesterol), and high $\mathrm{BP}$ define metabolic syndrome (MetS), a disorder of pandemic proportions (1-4). This constellation of risk factors with metabolic origin is associated with a substantially increased risk of developing type 2 diabetes, cardiovascular disease, and other life-threatening and debilitating disorders to a higher degree than any of these conditions individually $(2,3,5)$. As summarized in 2008, the prevalence of this complex disorder is between $20 \%$ and $30 \%$ of the adult population in the US and Canada, and many countries in Europe, Asia, and Latin America (4). Despite the critical need for treating MetS, no specific therapeutic approaches are currently available. Exercise and diet can potentially alleviate MetS, but there are numerous obstacles that preclude implementation of lifestyle modifications $(3,6)$. Pharmacological options are mostly directed to treating individual conditions (or risk factors) clustering in $\operatorname{MetS}(3,6)$. As previously noted, in addition to developing improved treatments for the individual components of MetS, it may be more efficient to also target major underlying pathophysiological events (4). Preclinical and

clinical studies have identified low-grade chronic inflammation, manifested by increased plasma levels of TNF and other proinflammatory cytokines and altered levels of adipokines (such as leptin and adiponectin), and insulin resistance, as important components of pathophysiological mechanisms underlying MetS $(3,6)$. Autonomic nervous system imbalance with increased indices of sympathetic activity and decreased vagus nerve activity has also been observed in MetS (7-11). A positive correlation between this autonomic dysfunction and the development of type 2 diabetes and cardiovascular disease has been established (12-14). Accordingly, lowering chronic inflammation, alleviating insulin resistance, and improving autonomic function have been proposed as therapeutic options $(11,15-17)$.

Vagus nerve activity has been implicated in the regulation of inflammation through a brain-integrated physiological mechanism termed the inflammatory reflex (18). Vagus nerve stimulation and activation of the inflammatory reflex have been successfully explored in animal and clinical studies of disorders characterized by inflammation, metabolic dysregulation, and autonomic dysfunction, including sepsis, inflammatory bowel disease, and rheumatoid arthritis (16, 19-21). The inflammatory reflex is also activated by galantamine, a centrally acting acetylcholinesterase inhibitor approved for the symptomatic treatment of Alzheimer's disease (22). Galantamine suppresses serum TNF and other proinflammatory cytokine levels and improves survival in lethal endotoxin-induced inflammation (23). Galantamine also lowers indices of colonic inflammation and alleviates disease severity in experimental inflammatory bowel disease (24). These antiinflammatory effects of galantamine are mediated through brain muscarinic acetylcholine receptor signaling and vagus nerve activity $(23,24)$. Galantamine administration to mice with high-fat diet-induced obesity and MetS significantly lowers plasma levels of proinflammatory cytokines and adipokines, and alleviates insulin resistance and other metabolic derangements (25). Accordingly, we reasoned that galantamine may be useful in alleviating inflammation in patients with MetS. 
Table 1. Baseline characteristics

\begin{tabular}{|c|c|c|c|c|c|c|c|}
\hline & \multicolumn{3}{|c|}{ Placebo Group } & \multicolumn{3}{|c|}{ Galantamine Group } & \multirow[b]{2}{*}{$P$ value } \\
\hline & $n=$ & \multicolumn{2}{|c|}{ mean (SD) } & $n=$ & \multicolumn{2}{|c|}{ mean (SD) } & \\
\hline \multicolumn{8}{|l|}{ Age and anthropometry } \\
\hline Height (m) & 30 & 1.65 & $(0.10)$ & 30 & 1.65 & $(0.08)$ & 0.923 \\
\hline Weight (kg) & 30 & 91.42 & $(13.06)$ & 30 & 93.19 & (11.88) & 0.304 \\
\hline BMI $\left(\mathrm{kg} / \mathrm{m}^{2}\right)$ & 30 & 33.75 & $(4.28)$ & 30 & 34.29 & $(3.55)$ & 0.584 \\
\hline Intra-abdominal fat tissue $\left(\mathrm{cm}^{2}\right)$ & 29 & 163.00 & (63.38) & 27 & 171.70 & (61.53) & 0.491 \\
\hline $\begin{array}{l}\text { Subcutaneous abdominal fat tissue } \\
\left(\mathrm{cm}^{2}\right)\end{array}$ & 29 & 375.79 & (119.48) & 27 & 364.22 & (108.89) & 0.646 \\
\hline Epicardial fat tissue (ml) & 29 & 168.67 & $(47.75)$ & 27 & 193.54 & $(66.01)$ & 0.158 \\
\hline Glucose (mg/dl) & 30 & 100.07 & (10.98) & 30 & 102.1 & $(10.76)$ & 0.549 \\
\hline Triglycerides (mg/dl) & 30 & 167.73 & $(72.83)$ & 30 & 181.07 & $(82.65)$ & 0.620 \\
\hline Total cholesterol (mg/dl) & 30 & 199.23 & $(45.69)$ & 30 & 198.07 & (39.02) & 0.994 \\
\hline \multicolumn{8}{|l|}{ BP and heart rate } \\
\hline SBP (mmHg) & 30 & 128.57 & $(13.97)$ & 30 & 123.20 & (9.9) & 0.164 \\
\hline $\mathrm{DBP}(\mathrm{mmHg})$ & 30 & 83.37 & $(9.32)$ & 30 & 79.47 & $(7.5)$ & 0.103 \\
\hline Ambulatory awake SBP (mmHg) & 30 & 125.77 & $(9.92)$ & 30 & 126.33 & $(12.77)$ & 0.882 \\
\hline Ambulatory awake DBP (mmHg) & 30 & 79.47 & $(8.41)$ & 30 & 81.60 & $(10.33)$ & 0.482 \\
\hline Ambulatory sleep SBP (mmHg) & 30 & 111.20 & $(12.47)$ & 30 & 111.57 & $(10.47)$ & 0.824 \\
\hline Ambulatory sleep DBP (mmHg) & 30 & 65.33 & $(10.25)$ & 30 & 66.73 & (9.93) & 0.514 \\
\hline Heart rate (bpm) & 30 & 70.57 & $(9.84)$ & 30 & 72.17 & $(9.11)$ & 0.351 \\
\hline \multicolumn{8}{|l|}{ Heart rate variability } \\
\hline IL-1及 (pg/ml) & 30 & 1.97 & $(1.7)$ & 30 & 4.2 & (6.38) & 0.617 \\
\hline IL-6 (pg/ml) & 30 & 5.37 & (9.29) & 30 & 6.05 & $(10.14)$ & 0.993 \\
\hline IL-10 (pg/ml) & 30 & 2.94 & $(2.04)$ & 30 & 4.81 & (11.63) & 0.408 \\
\hline Leptin (ng/ml) & 30 & 32.57 & $(21.42)$ & 28 & 33.34 & $(23.05)$ & 0.931 \\
\hline Adiponectin ( $\mu \mathrm{g} / \mathrm{ml})$ & 30 & 8.89 & $(1.58)$ & 30 & 8.88 & $(2.10)$ & 0.662 \\
\hline Leptin/adiponectin ratio & 30 & 3.78 & $(2.49)$ & 28 & 3.93 & $(2.48)$ & 0.821 \\
\hline
\end{tabular}

HOMA-IR, homeostasis model assessment of insulin resistance; SBP, systolic BP; DBP, diastolic BP; nu, normalized units; LF, low frequency; HF, high frequency.

The primary aim of this proof-of-concept study was to investigate the effect of a 12-week course of galantamine on plasma levels of TNF, IL-1 $\beta$, IL-6, IL-10, leptin, and adiponectin; cytokines and adipokines associated with the inflammatory state implicated in insulin resistance and cardio-metabolic derangements in patients with MetS. In addition, we examined effects of the drug on insulin resistance, autonomic regulation of heart function, and metabolic and cardiovascular indices.

\section{Results}

Patients. Between March 2013 and March 2015, 189 patients provided informed consent. After initial screening, and consideration of the inclusion and exclusion criteria, 60 eligible subjects ( 30 males and 30 females) were consecutively randomized in a 1:1 ratio to treatment with galantamine (15 males and 
Table 2. Effect of galantamine treatment on inflammatory markers

\begin{tabular}{|c|c|c|c|c|c|c|c|c|c|c|c|c|c|c|c|c|}
\hline \multirow{3}{*}{$\begin{array}{l}\text { Cytolkines and } \\
\text { Adipokines }\end{array}$} & \multicolumn{6}{|c|}{ Baseline } & \multicolumn{6}{|c|}{ Follow-up } & \multirow{2}{*}{\multicolumn{4}{|c|}{$\begin{array}{c}\text { Treatment } \times \text { Time Interaction } \\
\text { Effect }\end{array}$}} \\
\hline & \multicolumn{3}{|c|}{ Placebo Group } & \multicolumn{3}{|c|}{ Galantamine Group } & \multicolumn{3}{|c|}{ Placebo Group } & \multicolumn{3}{|c|}{ Galantamine Group } & & & & \\
\hline & $n=$ & mean & (SD) & $n=$ & mean & (SD) & $n=$ & mean & (SD) & $n=$ & mean & (SD) & Effect & $\left(95^{\circ}\right.$ & $\mathrm{Cl})$ & $P$ value \\
\hline $\mathrm{IL}-1 \beta(\mathrm{pg} / \mathrm{ml})$ & 30 & 1.97 & $(1.7)$ & 30 & 4.2 & (6.38) & 30 & 2.13 & $(2.65)$ & 30 & 2.46 & $(5.11)$ & -1.88 & -3.28 & -0.7 & 0.078 \\
\hline IL-6 (pg/ml) & 30 & 5.37 & (9.29) & 30 & 6.05 & (10.14) & 30 & 3.97 & $(4.16)$ & 30 & 4.76 & $(9.21)$ & 0.17 & -2.18 & 3.08 & 0.412 \\
\hline IL-10 (pg/ml) & 30 & 2.94 & $(2.04)$ & 30 & 4.81 & $(11.63)$ & 30 & 2.17 & $(1.6)$ & 30 & 5.33 & (13.34) & 1.32 & 0.29 & 2.38 & 0.002 \\
\hline $\begin{array}{l}\text { Leptin/ } \\
\text { adiponectin ratio }\end{array}$ & 30 & 3.78 & $(2.49)$ & 28 & 3.93 & $(2.48)$ & 30 & 5.33 & (3.29) & 28 & 2.81 & $(1.97)$ & -2.67 & -3.46 & -1.87 & $<0.0001$ \\
\hline
\end{tabular}

15 females) and placebo (15 males and 15 females). The trial profile is depicted in Figure 1. All enrolled patients completed the trial and none were lost to follow-up. Baseline characteristics of the study population are shown in Table 1 . The average age was 42.7 years and 40.8 years for the placebo and the galantamine groups, respectively. Baseline characteristics, including anthropometric indices, fat tissue depots, metabolic markers, BP, heart rate, heart rate variability (HRV) components, and cytokines and adipokines did not differ between the 2 groups (Table 1).

Effects of galantamine on primary outcomes. The effects of galantamine treatment on the primary outcome measures, plasma cytokines and adipokines, are presented in Table 2. At the end of the 12-week treatment period, plasma levels of the proinflammatory cytokine TNF decreased with galantamine treatment and this change was statistically significant compared with placebo $(-2.57 \mathrm{pg} / \mathrm{ml}[95 \% \mathrm{CI}$ -4.96 to -0.19 ]; $P=0.035)$. Galantamine also decreased plasma levels of IL-1 $\beta$, but this effect did not reach statistical significance compared with placebo $(-1.88 \mathrm{pg} / \mathrm{ml}[95 \% \mathrm{CI}-3.28$ to -0.7$] ; P=0.078)$. Galantamine had no significant effect on the plasma levels of IL-6 $(0.17 \mathrm{pg} / \mathrm{ml}$ [95\% CI -2.18 to 3.08]; $P=0.412$ ) compared with placebo. Conversely, galantamine treatment resulted in significantly increased plasma levels of the antiinflammatory cytokine IL-10 (1.32 pg/ml [95\% CI 0.29 to 2.38 ]; $P$ $=0.002$ ) compared with placebo. It should be noted that IL-1 $\beta$, IL-6, and IL-10 levels included values below the lower detectable limits of the assays. The frequency of these values below the lower detectable limit is shown in Table 3. These values were evenly distributed between groups and therefore should not have disproportionately affected the analyses.

Plasma adipokine levels also were altered by galantamine treatment. Plasma levels of leptin, a molecule with proinflammatory properties, were significantly decreased in patients taking galantamine $(-12.02$ ng/ml [95\% CI -17.71 to -6.33$] ; P<0.0001)$ compared with those taking placebo. In contrast, plasma levels of adiponectin, an antiinflammatory mediator, were significantly increased in the galantamine group $(2.71 \mu \mathrm{g} / \mathrm{ml}$ [95\% CI 1.93 to 3.49]; $P<0.0001)$ compared with the placebo group. Accordingly, the leptin/ adiponectin ratio was significantly decreased with galantamine treatment $(-2.67$ [95\% CI -3.46 to -1.87 ]; $P$ $<0.0001$ ) compared with placebo (Table 2).

Table 3. Frequency of results below the lower detectable limit for cytokine assays

\begin{tabular}{|c|c|c|c|c|}
\hline \multirow[t]{2}{*}{ Cytokine $^{A}$} & \multicolumn{2}{|c|}{ Pretreatment number of subjects (\%) } & \multicolumn{2}{|c|}{ Posttreatment number of subjects (\%) } \\
\hline & placebo & galantamine & placebo & galantamine \\
\hline IL-1 $\beta$ & $8(27 \%)$ & $10(33 \%)$ & $18(60 \%)$ & $17(57 \%)$ \\
\hline IL-6 & $12(40 \%)$ & $12(40 \%)$ & $13(43 \%)$ & $16(53 \%)$ \\
\hline IL-10 & $7(23 \%)$ & $10(33 \%)$ & $9(30 \%)$ & $10(33 \%)$ \\
\hline
\end{tabular}

AValues that were below the lower detectable limit of the assay were set to $0.79 \mathrm{pg} / \mathrm{ml}$ for IL-1 $\beta, 0.89 \mathrm{pg} / \mathrm{mL}$ for IL-6, and $1.09 \mathrm{pg} / \mathrm{ml}$ for IL-10 
Effects of galantamine on secondary outcomes. Galantamine effects on secondary outcome measures are shown in Table 4. Homeostasis model assessment of insulin resistance (HOMA-IR) was significantly lower in patients receiving galantamine (log scale 0.55 [95\% CI 0.36 to 0.85$] ; P=0.008$ ) compared with those on placebo. This drug effect was related to a significant decrease in insulin levels in the galantamine group (log scale $0.57 \mathrm{mU} / 1$ [95\% CI 0.37 to 0.87 ]; $P=0.010$ ) compared with the placebo group. Glucose levels were also decreased in the galantamine group $(-5.57 \mathrm{mg} / \mathrm{dl}[95 \% \mathrm{CI}-11.77$ to 0.63$] ; P=0.077)$, but this drug effect was not statistically significant. HRV frequency components reflecting autonomic modulation of the cardiac function were also altered by galantamine treatment (Table 4). The low frequency (LF) power $\left(\mathrm{ms}^{2}\right)$ of HRV in the galantamine group was significantly decreased, compared with the placebo group (log scale $0.48 \mathrm{~ms}^{2}$ [95\% CI 0.29 to 0.80$] ; P=0.005$ ). This alteration was associated with significantly decreased LF (normalized units [nu]) of HRV and a corresponding increase in high frequency (HF) (nu) of HRV, compared with placebo. Accordingly, a lower LF/HF ratio was determined in the galantamine arm (log scale $0.50 \mathrm{nu}$ [95\% CI 0.35 to 0.71$] ; P<0.0001$ ). Fat tissue depots in the galantamine and placebo groups did not differ significantly: intra-abdominal fat tissue $\left(-1.98 \mathrm{~cm}^{2}\right.$ [95\% CI -10.25 to 6.29]; $\left.P=0.633\right)$; subcutaneous abdominal fat tissue $\left(-0.29 \mathrm{~cm}^{2}[95 \% \mathrm{CI}-16.32\right.$ to 15.74$\left.] ; P=0.971\right)$; and epicardial fat tissue $(-14.99$ $\mathrm{ml}$ [95\% CI -33.64 to 3.65]; $P=0.113$ ) (Table 4). The spleen, an important organ target of the inflammatory reflex $(16)$ activated by galantamine $(23,24)$ was visualized while performing intra-abdominal fat tissue assessment. Thus, potential alterations in galantamine effects as a result of prior splenectomy were excluded. In parallel with the lack of effect of galantamine on fat depots, no significant differences between the groups were determined in body weight $(0.07 \mathrm{~kg}$ [95\% CI -1.26 to 1.39$] ; P=0.918), \mathrm{BMI}\left(0.02 \mathrm{~kg} / \mathrm{m}^{2}\right.$ [95\% CI -0.46 to 0.50$] ; P=0.949)$, and waist circumference $(-0.87 \mathrm{~cm}[95 \% \mathrm{CI}-2.35$ to 0.61$] ; P=0.245)$. HDL cholesterol levels were nonsignificantly increased $(0.88 \mathrm{mg} / \mathrm{dl}[95 \% \mathrm{CI}-2.19$ to 3.95$] ; P=0.568)$ and LDL cholesterol $(-1.73 \mathrm{mg} / \mathrm{dl}[95 \% \mathrm{CI}-15.78$ to 12.31$] ; P=0.805)$, triglyceride $(-8.2 \mathrm{mg} / \mathrm{dl}[95 \% \mathrm{CI}$ -38.81 to 22.42$] ; P=0.593)$, and total cholesterol levels $(-1.99 \mathrm{mg} / \mathrm{dl}[95 \% \mathrm{CI}-15.28$ to 11.29$] ; P=0.764)$ nonsignificantly decreased with galantamine treatment compared with placebo (Table 4 ). We used distinctive and complementary methods to determine arterial BP, considering its importance in physiology and pathophysiological conditions, including MetS. Evaluation of office BP registration, a common method used for clinical assessment, showed no significant effect of galantamine on the systolic BP (SBP) (2.47 $\mathrm{mmHg}$ [95\% CI -4.23 to 9.16]; $P=0.464)$ and diastolic BP (DBP) $(1.70 \mathrm{mmHg}$ [95\% CI -4.0 to 7.4$] ; P=$ $0.553)$ compared with placebo. Furthermore, ambulatory BP (ABP) monitoring (ABPM) revealed no significant differences in the awake SBP $(1.20 \mathrm{mmHg}[95 \% \mathrm{CI}-3.54$ to 5.94]; $P=0.614)$, awake DBP $(-1.07$ mmHg [95\% CI -4.63 to 2.50]; $P=0.551)$, sleep SBP (2.63 $\mathrm{mmHg}[95 \% \mathrm{CI}-3.38$ to 8.65$] ; P=0.551)$, and sleep DBP (0.43 mmHg [95\% CI -4.07 to 4.94]; $P=0.848)$ between the groups. Galantamine did not significantly alter heart rate $(1.74 \mathrm{bpm}[95 \% \mathrm{CI}-2.47$ to 5.95$]$; $P=0.411)$ compared with placebo (Table 4).

Adverse effects. Galantamine treatment was well tolerated; only 3 adverse events were reported in both groups, and were categorized as minor based on the Common Terminology Criteria for Adverse Events (CTCAE 4.03, 2010): mild (grade 1) or moderate intensity (grade 2). These adverse effects were of short duration and no hospitalization was necessary. In the placebo group one subject had a single episode of nausea and diarrhea (grade 1) and another subject had a skin infection (grade 2). Of note, the skin infection was mild, occurred 7 weeks prior to the posttreatment assessment, and was resolved within 3 days. Therefore, it would not have impacted the endpoints, including cytokine levels. In the galantamine group one subject complained of dizziness (grade 1).

\section{Discussion}

To our knowledge, this double-blind placebo-controlled study is the first clinical trial to assess the effects of galantamine, a clinically approved drug for Alzheimer's disease, in patients with MetS. The results of the study demonstrate that within 12 weeks galantamine inhibited inflammation and insulin resistance in association with alterations in autonomic tone.

Weight gain and abdominal obesity have been proposed as the main driving forces of metabolic dysregulation, inflammation, and insulin resistance in $\operatorname{MetS}(3,6)$. This notion is supported by observations that weight loss alleviates indices of these derangements $(6,26)$. Interestingly, weight loss does not always result in significant and uniform alleviation of the inflammatory state. For instance, in contrast to other markers, increased plasma TNF levels and decreased adiponectin levels in patients with MetS may not change after weight loss (26). In addition to numerous obstacles in achieving and sustaining weight loss, these find- 
Table 4. Effect of galantamine on secondary endpoints

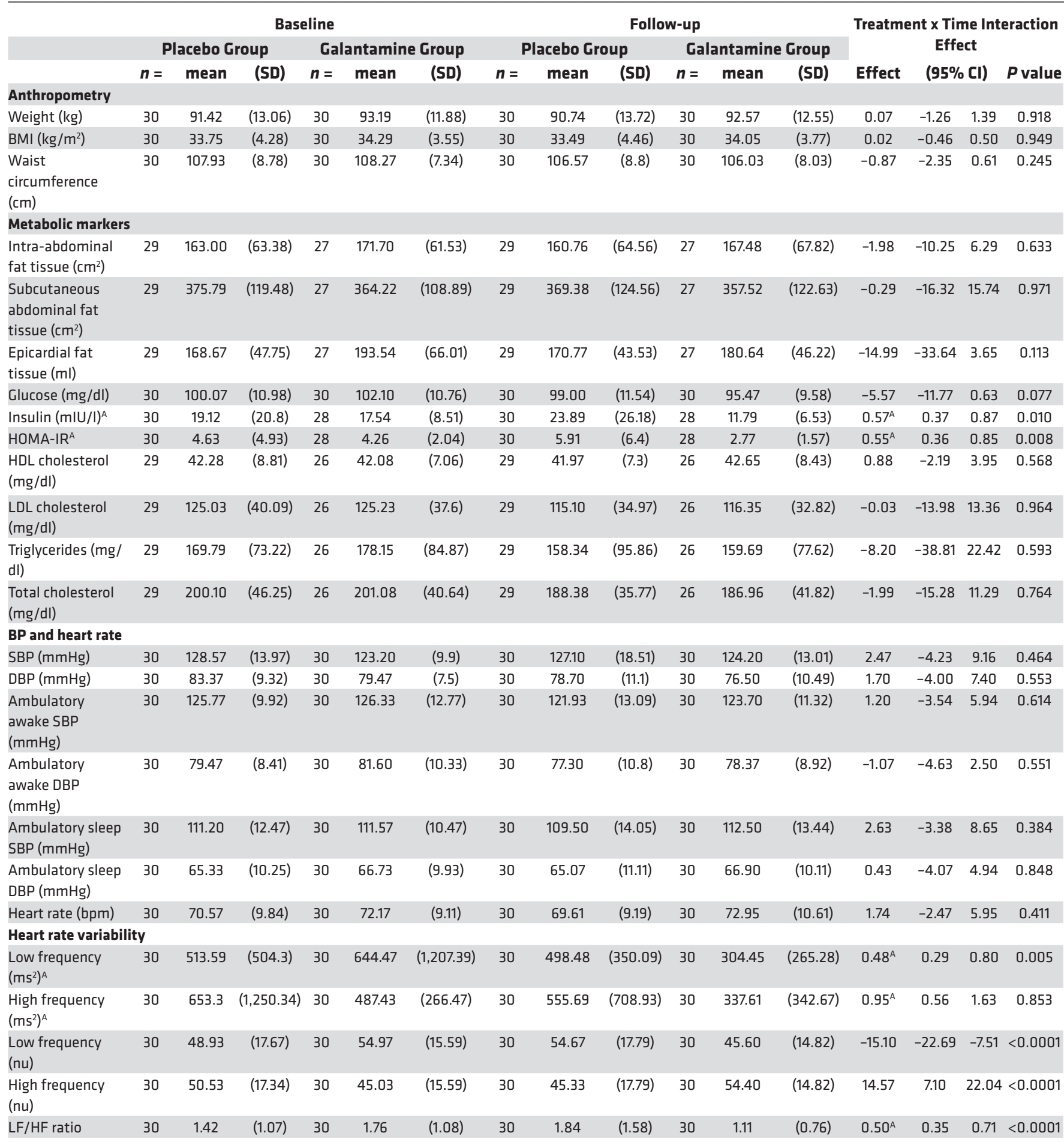

${ }^{A}$ These variables were analyzed on a logarithmic scale. HOMA-IR, homeostasis model assessment of insulin resistance; SBP, systolic BP; DBP, diastolic BP; nu, normalized units; LF, low frequency; HF, high frequency.

ings suggest the need of targeted interventions in MetS to aggressively manage the inflammatory state and lower the risk of type 2 diabetes and cardiovascular disease. To evaluate the impact of galantamine on the inflammatory state in MetS, we examined the effect of the drug on circulating levels of inflammatory molecules, including cytokines and adipokines, with a recognized role in insulin resistance and cardiovascular 
risk. TNF and IL-1 $\beta$ are prototypical proinflammatory cytokines, major contributors to insulin resistance $(6,16,17)$. TNF has been shown to induce insulin resistance, a discovery that directly linked inflammation to insulin resistance $(27,28)$. TNF also suppresses the expression of adiponectin (an important adipokine with antiinflammatory, insulin-sensitizing, and antiatherogenic properties) in human adipocytes (29). IL-1 $\beta$ impairs insulin signaling and action in adipocytes (30). Both TNF and IL-1 $\beta$ are potential therapeutic targets in MetS and type 2 diabetes $(15,16,31)$. IL-6 has also been implicated in insulin resistance and identified as a procoagulant factor $(16,17)$. The prototypical antiinflammatory cytokine IL-10 has been associated with insulin-sensitizing effects $(17,32)$. To our knowledge, despite the documented involvement of these pro- and antiinflammatory cytokines in MetS, thorough evaluation of their circulating levels in subjects with MetS has not been previously performed. Our results indicate that while plasma TNF levels in subjects with MetS can be determined, plasma levels of IL-1 $\beta$, IL-6, and IL-10 remain relatively low (below the lower detectable limit of a sensitive assay) in some individuals. Galantamine treatment had selective effects on circulating cytokine levels, manifested by reduced plasma TNF, no significant alterations in plasma IL-1 $\beta$ and IL-6, and increased plasma IL-10 as compared with placebo. Understandably, the drug effects on plasma IL-1 $\beta$, IL- 6 , and IL-10 should be interpreted in the context of the low circulating levels of these cytokines in MetS as noted above. The adipokine adiponectin is an antiinflammatory molecule, which also suppresses macrophage activation and the release of proinflammatory cytokines, including TNF $(29,33)$. Adiponectin whose levels are typically decreased in MetS has insulin-sensitizing and antiatherogenic functions $(6,16,29,33)$. Increased leptin levels (hyperleptinemia) in obesity and MetS are associated with proinflammatory effects $(6,16,33)$ and are linked with insulin resistance $(16,33)$, risk for thrombotic complications in obese individuals (34), and arterial distensibility (35). The leptin/adiponectin ratio is proposed as an indicator of insulin-resistant states and a preferential atherogenic marker as compared with leptin and adiponectin alone. Galantamine increased plasma adiponectin, decreased plasma leptin and the leptin/adiponectin ratio, and lowered insulin resistance (HOMA-IR). The observed lower plasma insulin levels in the galantamine group is of particular interest, because compensatory insulinemia has been identified as an independent predictor of ischemic heart disease (36) and type 2 diabetes (37) in individuals with MetS.

Previously, treating mice with obesity and MetS with a relatively high galantamine dose has been shown to significantly alleviate inflammation and insulin resistance in parallel with a moderate weight loss and reduced abdominal fat tissue depots (25). Therefore, the antiinflammatory effects of galantamine could, at least partially, be related to a weight loss effect of the drug in the animal model. In contrast to these preclinical observations, our findings here indicate antiinflammatory and insulin resistance-alleviating effects of lowdose galantamine treatment, which are not associated with significant weight loss, BMI, and fat tissue depot reduction in this relatively short 12 -week treatment period. Galantamine antiinflammatory effects have been previously linked to activation of the vagus nerve-based inflammatory reflex $(16,23,24)$. Stimulation of vagus nerve activity and galantamine treatment have been shown to result in alleviation of inflammation in preclinical settings of diseases characterized with metabolic derangement and autonomic dysfunction, including inflammatory bowel disease and arthritis $(16,38,39)$. In addition to vagus nerve cholinergic signaling, sympathetic catecholaminergic signals regulate inflammation $(16,21)$. Obesity and MetS are associated with autonomic dysfunction, and inhibiting sympathetic or enhancing vagus nerve activity have been suggested as therapeutic approaches $(11,16)$. HRV analysis is a noninvasive measure that provides information related to autonomic regulation and relatively limited insight into the balance between sympathetic and vagus nerve activity (40). Galantamine treatment altered the HRV frequency components and significantly lowered LF/ $\mathrm{HF}$ ratios. These findings suggest that potential mechanisms of the antiinflammatory and insulin resistancealleviating effects of galantamine may be related to its effects on autonomic regulation.

The highest galantamine dose clinically approved for the treatment of Alzheimer's disease is $24 \mathrm{mg}$ daily. A drug dose of $32 \mathrm{mg}$ daily has also been tested in clinical trials with Alzheimer's disease patients (22). In the present study, galantamine was dosed at $16 \mathrm{mg}$ daily after a dose escalation in individuals with much higher BMIs as compared with the typical Alzheimer's disease patient, and the short treatment period likely precluded significant changes in weight and abdominal circumference. Therefore, it may not be surprising that no significant drug effects on body weight, BMI, and fat tissue depots were found in our short study. In addition, no significant effects of galantamine on several metabolic markers, including HDL cholesterol, LDL cholesterol, total cholesterol, and triglycerides were determined. No significant alterations in heart rate and in office $\mathrm{BP}$ and $\mathrm{ABP}$ were observed as a result of galantamine treatment, which is 
consistent with a previous report in Alzheimer's disease patients (41). Intriguingly, treatments with galantamine and other centrally acting acetylcholinesterase inhibitors of Alzheimer's disease patients have been previously found to decrease the risk of myocardial infarction and death (42).

While Alzheimer's disease is a disorder of the elderly, MetS has no age boundaries and affects even children $(6,43)$. Autonomic nervous system dysfunction with decreased $\mathrm{HF}$ and increased LF/HF ratio of HRV have been reported in adolescents with MetS, in addition to metabolic derangements (43). Galantamine and other centrally acting acetylcholinesterase inhibitors have been clinically used in pediatric populations, for instance in children with autism spectrum disorder (44), where galantamine has repeatedly been found to improve core and associated symptoms of the disease $(44,45)$. Importantly, no significant difference in the frequency of side effects between the drug and the placebo arms has been found in a randomized, doubleblind, placebo-controlled study of the effects of galantamine (up to $24 \mathrm{mg} /$ day) for 10 weeks in children with autism, indicating a favorable safety drug profile (45) as we are reporting in this study. This previous clinical experience may be useful while designing studies investigating galantamine in pediatric MetS

MetS and type 2 diabetes can be frequently associated with cognitive deterioration (46). The importance of considering alleviation of cognitive problems in strategizing new therapeutic approaches for these conditions has been emphasized (47). Our results together with the previous extensive characterization of galantamine as a cognitive enhancer suggest a dual beneficial role of galantamine that should be considered in designing future studies with galantamine in MetS, type 2 diabetes, and other diseases stemming from MetS.

Our study has strengths and limitations. The study had many features of a good clinical trial design: parallel groups with equal sexual representation per group, concealed randomization, placebo control, and blinding of participants and investigators. The sample size of 30 participants per group provided ample power to detect meaningful differences between galantamine- and placebo-treated patients. The effects of galantamine were assessed using well-validated tests and assays. A failure in performing fat depot tissue assessment in a few patients (who did not come for this test) and technical errors related to transportation and processing of blood samples resulted in a limited number of missing data; these represent limitations of the study.

In conclusion, our results demonstrate that galantamine, a drug in clinical use for the symptomatic treatment of Alzheimer's disease, ameliorates important components of MetS pathogenesis, including inflammation and insulin resistance and alters autonomic tone. These effects were seen at a low galantamine dose and the drug was extremely well tolerated. Further studies to examine the predictive value of these favorable alterations in inflammation and insulin resistance in this at-risk population and the longterm clinical benefits of galantamine in the treatment of MetS should be pursued.

\section{Methods}

Study design and participants. This was a randomized, double-blind, placebo-controlled study of the effects of galantamine treatment in patients diagnosed with MetS. The study was performed at a clinical research center, The Heart Institute (InCor), University of São Paulo, Brazil. Study participants were recruited from outpatient settings. Individuals of both sexes between the ages of 18 and 59 years, and with BMI measures between 25 and $39 \mathrm{~kg} / \mathrm{m}^{2}$ were screened for the presence of MetS according to the ATP III criteria (1). These criteria require the presence of at least 3 of the following 5 parameters: increased abdominal circumference ( $\geq 102 \mathrm{~cm}$ for men and $\geq 88 \mathrm{~cm}$ for women); low plasma HDL cholesterol levels ( $<40 \mathrm{mg} / \mathrm{dl}$ for men and $<50 \mathrm{mg} / \mathrm{dl}$ for women); increased values for plasma triglycerides ( $\geq 150 \mathrm{mg} / \mathrm{dl})$; elevated BP $(\geq 130 \mathrm{~mm}$ $\mathrm{Hg}$ SBP or $\geq 85 \mathrm{~mm} \mathrm{Hg} \mathrm{DBP}$ ); and increased plasma glucose levels ( $\geq 100 \mathrm{mg} / \mathrm{dl}$ ). All subjects were evaluated to exclude conditions that restricted their inclusion in the study, which included symptoms and signs of cardiovascular disease or previous diagnosis of cardiac arrhythmias; coronary artery disease; valvular disease; heart failure; chronic obstructive pulmonary disease; chronic inflammatory diseases; cancer; positive status for HIV; abuse of alcohol or other illicit substances in the months prior to study entry; chronic use of medications, including drugs that have known or probable interaction with galantamine (serotonin reuptake inhibitors, amitriptyline, fluoxetine, fluvoxamine, ketoconazole, oxybutynin, paroxetine, quinidine); symptoms and signs of neurologic and autonomic diseases; past history of major depression, suicidal ideation, and history of eating disorders; triglyceride levels $400 \mathrm{mg} / \mathrm{dl}$ or higher; known history of liver disease or levels of aspartate transaminase (AST) or alanine transaminase (ALT) 200 U/1 or higher; office BP $160 \mathrm{mmHg}$ or higher or DBP $110 \mathrm{mmHg}$ or higher; and abnormal renal and thyroid function. All women were tested for pregnancy as an exclusion criterion. CONSORT 2010 guidelines (http://www. consort-statement.org) were followed during the preparation of this manuscript. 
Outcomes. The primary outcome measures of this study were plasma levels of cytokines and adipokines with a recognized role in mediating the MetS inflammatory state and linked to insulin resistance and cardio-metabolic derangements, including TNF, IL-1 $\beta$, IL-6, IL-10, leptin, and adiponectin. Secondary endpoints were fasting plasma glucose and insulin levels and the associated HOMA-IR, HRV, fat tissue depots (measured by computed tomography), metabolic and cardiovascular indices, including HDL cholesterol, LDL cholesterol, total cholesterol, triglycerides, arterial BP, and heart rate. No changes in the trial outcomes were made on the protocol after the trial commenced. We did, however, make some edits to the text initially posted on ClinicalTrials.gov to better reflect and summarize information in the protocol; cytokines and adipokines as primary outcome measures and metabolic and cardiovascular indices as secondary outcome measures were specifically listed.

Randomization, masking, and blinding. In order to reduce sex bias, subjects were stratified by sexual phenotype prior to being randomized to the galantamine or placebo arm. Recruitment of study participants took place until 30 men and 30 women were recruited to allow randomization in equally divided groups by sex. Study participants were randomly assigned to 1 of 2 parallel groups at a 1:1 ratio: 30 subjects ( 15 males and 15 females) were assigned to galantamine treatment and 30 subjects (15 males and 15 females) to placebo. A computer-generated random sequence method was used to allocate the participants to the corresponding groups. Randomized packages of placebo and active drug were numbered from 1 to 60 (http://graphpad.com/quickcalcs/randomize1/). Members of the staff of the Central Pharmacy that did not participate in the study conducted the randomization and were responsible for the drug delivery and inventory. Specific drug accountability included quantities dispensed/received (capsule counting) every month, serial numbers, expiration date, and drug code number. To achieve masking (blinding), galantamine capsules were reencapsulated at the Central Pharmacy of the Clinical Hospital, Medical School of University of São Paulo. Active drug and placebo capsules were presented in identical medication packaging. The study drug packages were stored in a secure, temperature-controlled medication room according to standard operating procedures. Subjects received packages of study drug for $30 \pm 3$ days, every 4 weeks for a total of 12 weeks. Study participants, investigators, and outcome assessors were blinded to the group assignment.

Study procedures. Galantamine hydrobromide extended-release capsules of $8 \mathrm{mg}$ and $16 \mathrm{mg}$, commercially available as Reminyl ER (manufactured by Janssen-Cilag Pharmaceuticals, Johnson \& Johnson) or placebo were used in the study. Galantamine was administered orally in a dose of $8 \mathrm{mg}$ for 4 weeks, and then titrated to $16 \mathrm{mg}$ for 8 weeks, for a total of 12 weeks. Following informed consent, at the initial screening all subjects underwent complete clinical and neurological evaluations including BP, waist circumference, height, weight, and BMI. Blood tests, including triglycerides, total cholesterol, HDL cholesterol and LDL cholesterol, fasting glucose, serum creatinine, thyroid and liver function tests, renal function panel, pregnancy testing (if applicable), and complete blood count were performed. Electrocardiogram and chest $\mathrm{x}$-ray were also performed. Based on results from the screening visit, eligible subjects (fulfilling the criteria for enrollment in the study) returned 7-10 days later for additional assessments that included determination of intra-abdominal, subcutaneous abdominal, and epicardial fat tissue depots, heart rate, office and 24-hour ABPM, HRV analysis, blood tests for plasma cytokine, adipokine and insulin levels, and HOMAIR assessment. After completing all evaluations, subjects were randomized and instructed to start taking the study drug. All subjects were assessed at 4 and 8 weeks for safety monitoring with clinical and neurological exams. After 12 weeks, at the end of the treatment period, subjects repeated the specific assays for fat tissue depot determination, heart rate determination, office and 24-hour ABPM, HRV analysis, blood tests and plasma cytokine, adipokine and insulin determination, and HOMA-IR assessment. The principal investigator, coinvestigators, and the principal coordinator monitored all study participants who were also able to communicate with the investigators by phone or email if necessary. Subject self-reporting of medication adherence and capsule counts were used to establish treatment compliance.

Methods and assays. Intra-abdominal (visceral) and subcutaneous abdominal fat were determined by computed tomography scans at the Cardiac Tomography and Resonance Service Center at the same hospital. Briefly, intra-abdominal fat and subcutaneous abdominal fat were measured by computed tomography (Aquilion One, Toshiba Medical Systems) at the level of the 4th and 5th (L4 and L5) lumbar vertebrae. All study participants were examined in the supine position with both arms stretched above the head, and 10-mm slices were measured. Intra-abdominal and subcutaneous abdominal fat areas $\left(\mathrm{cm}^{2}\right)$ were obtained by delineating and computing the adipose tissue surface using attenuation range of -150 
to -50 Hounsfield units (HU) (48). For analysis an automated selection of visceral fat area was taken by using threshold technique in the range of -30 to $-190 \mathrm{HU}$ with subsequent volume calculation. For this analysis, we used a workstation with Aquarius Intuition software (Terarecon), which automatically separates visceral abdominal fat from subcutaneous fat, and exports the corresponding volumes of the 2 compartments. To evaluate the total chest fat around the heart, a Vitrea workstation (Vital Images) was used with a volumetric threshold instrument defining fat as voxels with a tissue density between -30 and $-190 \mathrm{HU}$. Epicardial fat was defined as the one internal to the pericardium and in direct contact with the epicardial surface of the heart. This assessment was performed using the Vitrea workstation volumetric tool with manual planimetry, drawing a dividing line at the pericardium. Thus, average density and volume (in ml) of the epicardial compartment was generated.

Twenty-four-hour ABPM was performed using a SpaceLabs healthcare device (model 90207). BP was measured every 10 minutes during the day ( 8 AM to $11 \mathrm{PM}$ ) and every 20 minutes during the night (11 PM to $8 \mathrm{AM}$ ) with an appropriate cuff placed on a nondominant arm. Participants were instructed to perform their ordinary daily activities and not to move their arm during the ongoing measurement. Activity, bedtime, and time on awakening from sleep were recorded by participants in their diaries. Therefore, daytime and nighttime BP based on 24-hour ABPM was evaluated using actual sleep and wake times recorded by participants.

For HRV analysis all subjects were asked to abstain from exercise 24 hours before the study and from drinking caffeinated products on the morning of the test. The test was performed in a quiet room. BP waveforms were obtained with a digital photoplethysmograph device (Finometer; Finapres Medical System BV) while subjects were awake in a supine position during a 15-minute rest period. These stored data were subsequently analyzed to calculate HRV values. All of the segments were visually inspected, and the nonstationary data were discarded. A Hanning window was used to attenuate the side effects, and the spectrum of each segment was computed using a direct fast Fourier transform (FFT). Spectral bands were defined according to literature references of evaluation in humans: LF (0.04-0.15 Hz), HF (0.15-0.4 Hz), and total power. Spectral components were expressed in absolute values $\left(\mathrm{ms}^{2}\right)$ and nu. The nu were obtained by calculating the percentage of LF and HF with respect to the total power after subtracting the power of the very-low-frequency component (frequencies of $<0.04 \mathrm{~Hz}$ ). To assess the sympathovagal balance, the LF/HF ratio of the HRV was calculated (49, 50).

For plasma cytokine, adipokine, and insulin determination venous blood was collected from the participants and plasma samples obtained. Samples were stored at $-80^{\circ} \mathrm{C}$ prior to analysis. Cytokines, adipokines, and insulin were analyzed using multiplex immunoassay (all from Millipore): HCYTMAG-60K-PX41 for TNF, IL-1 $\beta$, IL-6, and IL-10; HADK2MAG-61K for leptin and insulin; and HADK1MAG-61K for adiponectin. Individual values for leptin and adiponectin were used to calculate the leptin/adiponectin ratio.

Statistics. The proposed sample size for this trial was 60 subjects ( $n=30$ per group), based on resources available and in consistency with previous clinical studies $(51,52)$. It should be noted that a sample size of 30 in each group will have $86 \%$ power to detect an effect size of 0.8 using a 2-group $t$ test with a 0.05 2 -sided significance level. This is consistent with the power analysis previously used in studying the effects of a drug (rosiglitazone) or placebo once daily for 12 weeks on inflammatory and metabolic indices in 60 patients with the metabolic syndrome (51).

Descriptive statistics for all subjects at baseline are reported as means and SD separately by group (Table 1). The Mann-Whitney test was used to determine whether the difference for each of the baseline measures between the placebo and the galantamine groups was significant. As cited below, some variables were analyzed with a log transformation; however, these variables are summarized in their original units of measurement for the columns labeled "Baseline" and "Follow-up" in Tables 1, 2, and 4. For the columns labeled "Treatment $\times$ Time Interaction Effect", they are summarized in the log scale.

Repeated measures analysis of variance (RMANOVA) with a mixed models approach was used to determine if the 2 groups behaved differently over time (pre- vs. post-) for most primary and secondary outcomes/endpoints (i.e., the group $\times$ time interaction). For all analyses, the standard assumptions of Gaussian residuals and equality of variance were tested. As the normality assumption was not met for insulin, HOMA-IR, high frequency $\left(\mathrm{ms}^{2}\right)$ of $\mathrm{HRV}, \mathrm{LF}\left(\mathrm{ms}^{2}\right)$ of $\mathrm{HRV}$, and LF/HF ratio, the logarithmic transformation was used for the analysis of these variables. The repeated-within-subjects factor was time and the within-subjects factor was treatment group (galantamine or placebo).

RMANOVA analyses were restricted to those subjects who had measurements at both baseline and follow-up visits. Means and SDs were reported at each time point for each of the 2 randomized treatment 
arms (Tables 2 and 4). The treatment effect sizes are presented as the arithmetic differences in least-squares means, with corresponding $95 \%$ CIs.

IL-1 $\beta$, IL-6, and IL-10 were subject to lower detectable limits $(0.8 \mathrm{pg} / \mathrm{ml}, 0.9 \mathrm{pg} / \mathrm{ml}$, and $1.1 \mathrm{pg} / \mathrm{ml}$, respectively). Therefore, any values that were below the lower detectable limit were set to $0.79 \mathrm{pg} / \mathrm{ml}, 0.89$ $\mathrm{pg} / \mathrm{ml}$, and $1.09 \mathrm{pg} / \mathrm{ml}$, respectively, for analysis (Table 3). Since this approach might render the required assumptions of the mixed-model analyses to be invalid, the bootstrap method using percentiles was used to estimate $95 \%$ CIs for the mean treatment $\times$ time interaction effect. The Mann-Whitney test was used to determine whether the difference in differences (Post-Pre) for each of these measures between the galantamine and placebo groups was significant.

A result was considered statistically significant at $P$ less than 0.05 . All analyses were performed using SAS version 9.4 (SAS Institute Inc.).

Study approval. The study was performed at The Heart Institute (InCor), University of São Paulo, Brazil. The study protocol was reviewed and approved by the Institutional Review Committee and the Human Subject Protection Committee of the Heart Institute (InCor) and the Clinic Hospital (number 11672/555738), University of São Paulo. Informed consent was obtained from all study participants prior to inclusion in the study. The study was conducted in accordance with World Medical Association International Code of Medical Ethics (Declaration of Helsinki, 1964; revised in 2008). The study is registered at www.clinicaltrials.gov with the number NCT02283242 and the following name: "Galantamine effects in patients with metabolic syndrome (GALANTA-MS)": https://clinicaltrials.gov/ct2/show/NCT02283242 ?term=galantamine+metabolic+syndrome\&rank $=1$.

Adverse effects monitoring and assessment. Prior placebo-controlled clinical trials with galantamine doses of $24 \mathrm{mg} /$ day and $32 \mathrm{mg} /$ day in patients with Alzheimer's disease have indicated that the most common adverse effects of these galantamine doses are gastrointestinal, particularly nausea (22). However, using a lower galantamine dose or slowly escalating the drug dose has been shown to substantially reduce the frequency of gastrointestinal adverse effects, thus demonstrating a favorable tolerability profile of galantamine (53). Therefore, in this study we implemented a regimen of low-dose galantamine treatment in which the initial drug dose ( $8 \mathrm{mg}$ /day) for 4 weeks was subsequently increased to $16 \mathrm{mg} /$ day for the remaining period of the study. The patients were informed about possible adverse effects and were encouraged to write down (document) and report any complaints and adverse effects. In addition, the patients were allowed and encouraged to report any adverse effect through unrestricted telephone contact with the investigator. Hospital support was available for the patients. To additionally ensure safety, clinical consultations were performed monthly and the participants in the study were asked about the occurrence of adverse effects, including nausea, vomiting, dizziness, diarrhea, abdominal pain, and tremor.

\section{Author contributions}

VAP, SKS, KJT, MA, and FMCC designed the study. CTS, FOC, TLM, HFL, JMM, MCI, CER, and LAB acquired the data. MA and MLL performed the statistical analyses. VAP, FMCC, DPB, MM, and KJT interpreted the data. VAP wrote the first draft of the report with inputs from FMCC, KJT, MA, and MLL. FMCC, CTS, HFL, YTH, SKS, PSO, MA, SSC, MM, DPB, MLL, JR, KJT, and VAP provided comments, participated in additional discussions, and revised the paper. All authors approved the final version.

\section{Acknowledgments}

This study was supported by Fundação de Amparo a Pesquisa do Estado de São Paulo (FAPESP: 2013/22250-9 (to FMCC) and Conselho Nacional de Desenvolvimento Científico e Tecnológico (CNPq), Brazil, and the following grants from the National Institute of General Medical Sciences, NIH: R01GM057226 (to KJT) and R01GM089807 (to KJT and VAP).

Address correspondence to: Valentin A. Pavlov, Center for Biomedical Science, The Feinstein Institute for Medical Research, 350 Community Dr., Manhasset, New York 11030, USA. Phone: 516.562.2316; Email: vpavlov@northwell.edu.

1. Grundy SM, et al. Diagnosis and management of the metabolic syndrome: an American Heart Association/National Heart, Lung, and Blood Institute Scientific Statement. Circulation. 2005;112(17):2735-2752.

2. Alberti KG, et al. Harmonizing the metabolic syndrome: a joint interim statement of the International Diabetes Federation 
Task Force on Epidemiology and Prevention; National Heart, Lung, and Blood Institute; American Heart Association; World Heart Federation; International Atherosclerosis Society; and International Association for the Study of Obesity. Circulation. 2009;120(16):1640-1645.

3. Cornier MA, et al. The metabolic syndrome. Endocr Rev. 2008;29(7):777-822.

4. Grundy SM. Metabolic syndrome pandemic. Arterioscler Thromb Vasc Biol. 2008;28(4):629-636.

5. McNeill AM, et al. The metabolic syndrome and 11-year risk of incident cardiovascular disease in the atherosclerosis risk in communities study. Diabetes Care. 2005;28(2):385-390.

6. Eckel RH, Grundy SM, Zimmet PZ. The metabolic syndrome. Lancet. 2005;365(9468):1415-1428.

7. Lambert GW, Straznicky NE, Lambert EA, Dixon JB, Schlaich MP. Sympathetic nervous activation in obesity and the metabolic syndrome--causes, consequences and therapeutic implications. Pharmacol Ther. 2010;126(2):159-172.

8. Assoumou HG, et al. Metabolic syndrome and short-term and long-term heart rate variability in elderly free of clinical cardiovascular disease: the PROOF study. Rejuvenation Res. 2010;13(6):653-663.

9. Licht CM, et al. Increased sympathetic and decreased parasympathetic activity rather than changes in hypothalamic-pituitaryadrenal axis activity is associated with metabolic abnormalities. J Clin Endocrinol Metab. 2010;95(5):2458-2466.

10. Karason K, Mølgaard H, Wikstrand J, Sjöström L. Heart rate variability in obesity and the effect of weight loss. Am J Cardiol. 1999;83(8):1242-1247.

11. Schlaich M, Straznicky N, Lambert E, Lambert G. Metabolic syndrome: a sympathetic disease? Lancet Diabetes Endocrinol. 2015;3(2):148-157.

12. Carnethon MR, et al. The association among autonomic nervous system function, incident diabetes, and intervention arm in the Diabetes Prevention Program. Diabetes Care. 2006;29(4):914-919.

13. Carnethon MR, Jacobs DR, Sidney S, Liu K, CARDIA study. Influence of autonomic nervous system dysfunction on the development of type 2 diabetes: the CARDIA study. Diabetes Care. 2003;26(11):3035-3041.

14. Thayer JF, Yamamoto SS, Brosschot JF. The relationship of autonomic imbalance, heart rate variability and cardiovascular disease risk factors. Int J Cardiol. 2010;141(2):122-131.

15. Esser N, Paquot N, Scheen AJ. Anti-inflammatory agents to treat or prevent type 2 diabetes, metabolic syndrome and cardiovascular disease. Expert Opin Investig Drugs. 2015;24(3):283-307.

16. Pavlov VA, Tracey KJ. The vagus nerve and the inflammatory reflex--linking immunity and metabolism. Nat Rev Endocrinol. 2012;8(12):743-754.

17. Shoelson SE, Herrero L, Naaz A. Obesity, inflammation, and insulin resistance. Gastroenterology. 2007;132(6):2169-2180.

18. Tracey KJ. The inflammatory reflex. Nature. 2002;420(6917):853-859.

19. Bonaz B, et al. Chronic vagus nerve stimulation in Crohn's disease: a 6-month follow-up pilot study. Neurogastroenterol Motil. 2016;28(6):948-953.

20. Koopman FA, et al. Vagus nerve stimulation inhibits cytokine production and attenuates disease severity in rheumatoid arthritis. Proc Natl Acad Sci U S A. 2016;113(29):8284-8289.

21. Pavlov VA, Tracey KJ. Neural regulation of immunity: molecular mechanisms and clinical translation. Nat Neurosci. 2017;20(2):156-166.

22. Raskind MA, Peskind ER, Wessel T, Yuan W. Galantamine in AD: A 6-month randomized, placebo-controlled trial with a 6-month extension. The Galantamine USA-1 Study Group. Neurology. 2000;54(12):2261-2268.

23. Pavlov VA, et al. Brain acetylcholinesterase activity controls systemic cytokine levels through the cholinergic anti-inflammatory pathway. Brain Behav Immun. 2009;23(1):41-45.

24. Ji H, Rabbi MF, Labis B, Pavlov VA, Tracey KJ, Ghia JE. Central cholinergic activation of a vagus nerve-to-spleen circuit alleviates experimental colitis. Mucosal Immunol. 2014;7(2):335-347.

25. Satapathy SK, et al. Galantamine alleviates inflammation and other obesity-associated complications in high-fat diet-fed mice. Mol Med. 2011;17(7-8):599-606.

26. Xydakis AM, et al. Adiponectin, inflammation, and the expression of the metabolic syndrome in obese individuals: the impact of rapid weight loss through caloric restriction. J Clin Endocrinol Metab. 2004;89(6):2697-2703.

27. Hotamisligil GS, Shargill NS, Spiegelman BM. Adipose expression of tumor necrosis factor-alpha: direct role in obesity-linked insulin resistance. Science. 1993;259(5091):87-91.

28. Feinstein R, Kanety H, Papa MZ, Lunenfeld B, Karasik A. Tumor necrosis factor-alpha suppresses insulin-induced tyrosine phosphorylation of insulin receptor and its substrates. J Biol Chem. 1993;268(35):26055-26058.

29. Ouchi N, Parker JL, Lugus JJ, Walsh K. Adipokines in inflammation and metabolic disease. Nat Rev Immunol. 2011;11(2):85-97.

30. Jager J, Grémeaux T, Cormont M, Le Marchand-Brustel Y, Tanti JF. Interleukin-1beta-induced insulin resistance in adipocytes through down-regulation of insulin receptor substrate-1 expression. Endocrinology. 2007;148(1):241-251.

31. Maedler K, Dharmadhikari G, Schumann DM, Storling J. Interleukin-targeted therapy for metabolic syndrome and type 2 diabetes. Handb Exp Pharmacol. 2011;(203):257-278.

32. van Exel E, et al. Low production capacity of interleukin-10 associates with the metabolic syndrome and type 2 diabetes : the Leiden 85-Plus Study. Diabetes. 2002;51(4):1088-1092.

33. Tilg H, Moschen AR. Adipocytokines: mediators linking adipose tissue, inflammation and immunity. Nat Rev Immunol. 2006;6(10):772-783

34. Konstantinides S, Schäfer K, Koschnick S, Loskutoff DJ. Leptin-dependent platelet aggregation and arterial thrombosis suggests a mechanism for atherothrombotic disease in obesity. J Clin Invest. 2001;108(10):1533-1540.

35. Singhal A, et al. Influence of leptin on arterial distensibility: a novel link between obesity and cardiovascular disease? Circulation. 2002;106(15):1919-1924.

36. Després JP, et al. Hyperinsulinemia as an independent risk factor for ischemic heart disease. N Engl J Med. 1996;334(15):952-957.

37. Hanson RL, Imperatore G, Bennett PH, Knowler WC. Components of the "metabolic syndrome" and incidence of type 2 diabetes. Diabetes. 2002;51(10):3120-3127.

38. Pavlov VA, Tracey KJ. Neural circuitry and immunity. Immunol Res. 2015;63(1-3):38-57.

39. Tracey KJ. Physiology and immunology of the cholinergic antiinflammatory pathway. J Clin Invest. 2007;117(2):289-296. 
40. Malliani A, Pagani M, Lombardi F, Cerutti S. Cardiovascular neural regulation explored in the frequency domain. Circulation. $1991 ; 84(2): 482-492$

41. Isik AT, Bozoglu E, Naharci MI, Kilic S. Evaluation of the effects of galantamine on cardiac function in elderly patients with Alzheimer's disease. Am J Geriatr Pharmacother. 2010;8(5):454-459.

42. Nordström P, Religa D, Wimo A, Winblad B, Eriksdotter M. The use of cholinesterase inhibitors and the risk of myocardial infarction and death: a nationwide cohort study in subjects with Alzheimer's disease. Eur Heart J. 2013;34(33):2585-2591.

43. Rodríguez-Colón SM, et al. Metabolic syndrome burden in apparently healthy adolescents is adversely associated with cardiac autonomic modulation--Penn State Children Cohort. Metab Clin Exp. 2015;64(5):626-632.

44. Rossignol DA, Frye RE. The use of medications approved for Alzheimer's disease in autism spectrum disorder: a systematic review. Front Pediatr. 2014;2:87.

45. Ghaleiha A, et al. Galantamine efficacy and tolerability as an augmentative therapy in autistic children: A randomized, doubleblind, placebo-controlled trial. J Psychopharmacol (Oxford). 2014;28(7):677-685.

46. Biessels GJ, Strachan MW, Visseren FL, Kappelle LJ, Whitmer RA. Dementia and cognitive decline in type 2 diabetes and prediabetic stages: towards targeted interventions. Lancet Diabetes Endocrinol. 2014;2(3):246-255.

47. Strachan MW, Reynolds RM, Marioni RE, Price JF. Cognitive function, dementia and type 2 diabetes mellitus in the elderly. Nat Rev Endocrinol. 2011;7(2):108-114.

48. Harada $\mathrm{PH}$, et al. Pericardial fat is associated with coronary artery calcification in non-dialysis dependent chronic kidney disease patients. PLoS One. 2014;9(12):e114358

49. [No authors listed]. Heart rate variability: standards of measurement, physiological interpretation and clinical use. Task Force of the European Society of Cardiology and the North American Society of Pacing and Electrophysiology. Circulation. 1996;93(5):1043-1065.

50. Atala MM, et al. Arg16Gly and Gln27Glu $\beta 2$ adrenergic polymorphisms influence cardiac autonomic modulation and baroreflex sensitivity in healthy young Brazilians. Am J Transl Res. 2015;7(1):153-161.

51. Samaha FF, et al. Effects of rosiglitazone on lipids, adipokines, and inflammatory markers in nondiabetic patients with low high-density lipoprotein cholesterol and metabolic syndrome. Arterioscler Thromb Vasc Biol. 2006;26(3):624-630.

52. Risérus U, Arner P, Brismar K, Vessby B. Treatment with dietary trans10cis12 conjugated linoleic acid causes isomer-specific insulin resistance in obese men with the metabolic syndrome. Diabetes Care. 2002;25(9):1516-1521.

53. Tariot PN, Solomon PR, Morris JC, Kershaw P, Lilienfeld S, Ding C. A 5-month, randomized, placebo-controlled trial of galantamine in AD. The Galantamine USA-10 Study Group. Neurology. 2000;54(12):2269-2276. 